\title{
Monitoring of hydrocarbon vapor diffusion in air using a thermal wave interferometer
}

\author{
J. A. P. Lima, M. G. da Silva, M. S. O. Massunaga, E. Marín, ${ }^{a}$ \\ and $\mathrm{H}$. Vargas ${ }^{\mathrm{b})}$ \\ Centro de Ciencia e Tecnología, Universidade Estadual do Norte Fluminense, Av Alberto Lamego 2000, \\ Campos dos Goytacazes, RJ 28015-620, Brazil \\ L. C. M. Miranda \\ Departamento de Física, Universidade Estadual de Maringá, 87020-900, Maringá, PR, Brazil
}

(Received 13 August 2001; accepted for publication 5 February 2002)

\begin{abstract}
A discussion on the use of thermal wave interference (TWI) for the monitoring of the transients of hydrocarbon in air is presented. The thermal wave signal was modeled using the logarithm-mixing model for the thermal diffusivity of a two-phase gas system in which the hydrocarbon vapor concentration in the air-filled TWI cell is a varying function of time. The time varying hydrocarbon vapor concentration was described assuming the simple Fick's model for mass diffusion of the hydrocarbon vapor in the stagnant air column of the TWI cell. The transient TWI signal amplitude data fitting yielded two parameters, namely, the saturation concentration and the characteristic diffusion time. From the corresponding values of the diffusion time the hydrocarbon mass diffusivities were straightforwardly obtained. The obtained values for the hydrocarbon mass diffusivities were found to be in good agreement with the ones reported in the literature. (c) 2002 American Institute of Physics. [DOI: 10.1063/1.1465104]
\end{abstract}

\section{INTRODUCTION}

The concept of thermal wave interference introduced by Bennett and Patty ${ }^{1}$ in the early 1980s has gained considerable interest in recent years after Shen and Mandelis ${ }^{2}$ have demonstrated feasibility of the pyroelectric detection of a thermal wave propagating across the air gap formed between a pyroelectric sensor and a thin aluminum-film wall acting as the source of thermal waves. The main attractive aspect of this new photothermal technique, originally called thermalwave resonant cavity, and later, thermal wave interferometer (TWI) by some other authors, is that it is well suited for the investigation of the thermophysical properties of gases and liquids. This can be appreciated by the growing number of papers that have recently applied this new technique to the investigation of the thermal properties of gases $^{3-6}$ and liquids. ${ }^{7}$ The reason the TWI technique is so attractive to the investigation of gases and liquids is evident from its operational principle, namely, the monitoring of the spatial dependence of intracavity thermal waves. The basic design of the TWI cavity consists of two walls separated by a gap of variable length $L$. One of the walls is a thermal wave generator and the other one is a temperature-sensing wall. The thermal wave generator consists of a thin aluminum foil on which a modulated light beam impinges on its black-painted outer surface, acting, accordingly, as a light absorber. Following the absorption of the modulated light beam, the Al foil temperature fluctuates periodically at the modulation frequency

\footnotetext{
${ }^{a}$ Permanent address: Facultad de Física, Universidad de La Habana, San Lázaro y L, Vedado 10400, Ciudad de La Habana, Cuba.

b) Author to whom correspondence should be addressed; electronic mail: vargas@uenf.br
}

of the incident beam thereby launching thermal waves into the gap between the two walls. The thermal waves thus generated propagate back and forth between the $\mathrm{Al}$ foil and the sensing wall, which consists of a thin-film pyroelectric sensor. The TWI method consists essentially of recording the temperature fluctuation of the pyroelectric sensor as a function of the cavity gap length. That is, the technique is essentially based upon measuring the temperature fluctuation on a sample of variable thickness. This fact renders this technique well suited for gas and liquid samples analysis.

One of the immediate applications of the TWI technique aimed the evaluation of the thermal diffusivity of air:hydrocarbon vapor mixtures. ${ }^{4-6}$ In these experiments a liquid portion of the hydrocarbon under investigation is placed inside the cell in which the initially air-filled TWI cavity is assembled. The hydrocarbon vapor resulting from the evaporation process diffuses into the air inside the TWI cavity. As a result of this, the thermal properties of the resulting gas mixture change with time, following the changes of the vapor concentration in the air. This process evolves with time until the hydrocarbon vapor inside the cell reaches saturation. Once saturation is reached, one then begins recording the TWI as a function of the cavity length in order to measure the thermal diffusivity of the resulting air:hydrocarbon vapor mixture. In this article we address ourselves to the quantitative understanding of the transient TWI data of air: hydrocarbon vapor mixtures.

\section{METHODOLOGY}

The TWI experimental setup has been discussed in detail elsewhere and it is schematically shown in Fig. 1. It consists of a temperature-controlled closed glass cell, adequately 


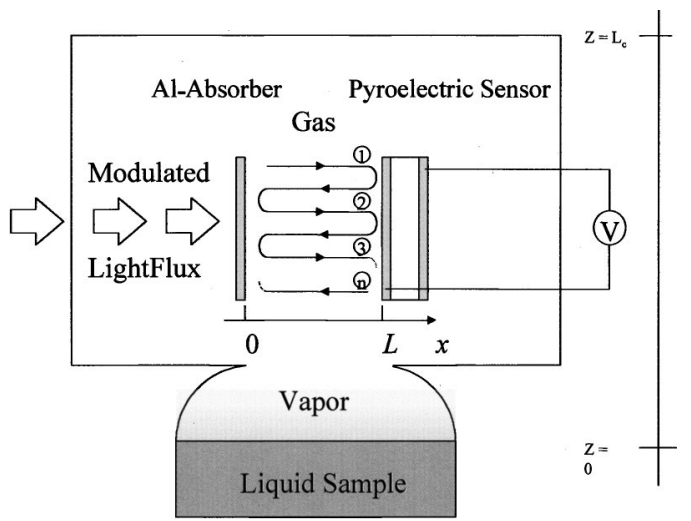

FIG. 1. Schematic view of the experimental TWI setup.

adapted for gas exchange and control of ambient parameters, in which the TWI is enclosed. At the bottom of the glass cell there is a screwed reservoir of $30 \mathrm{~mm}$ diameter on which 18 $\mathrm{ml}$ of the liquid hydrocarbon is poured. The vapors resulting from the hydrocarbon evaporation at ambient temperature diffuses into the air cell, filling the TWI cavity with the resulting air: hydrocarbon vapor mixture. The TWI cavity of variable length is formed between a $15 \mu \mathrm{m}$ thick $\mathrm{Al}$ foil and a pyroelectric sensor consisting of a $25 \mu \mathrm{m}$ thick polyvinyledene difluoride (PVDF) film with Al metalized surfaces. The $5 \mathrm{~mm}$ diameter $\mathrm{Al}$ foil and PVDF sensor were both cut from commercial sheets and inserted into two glass tubes soldered at the lateral ends of the glass cell. The left glass tube supports the $\mathrm{Al}$ foil that is bonded to the tube using epoxy glue. The surface of this $\mathrm{Al}$ foil facing the inner glass tube space is painted with black ink so that it acts as a light absorber. The second glass tube attached to the right glass cell end guides a nylon cylinder on top of which the pyroelectric sensor is assembled. This nylon cylinder is coupled to a translational stage so that the distance $L$ between the $\mathrm{Al}$ foil and the pyroelectric sensor can be varied. The vertical distance between the center of the resulting TWI cavity and the liquid hydrocarbon level at the bottom reservoir is equal to $7 \mathrm{~cm}$, whereas the vertical air column extending from the liquid level to the top of the glass cell is equal to $10 \mathrm{~cm}$.

The $5 \mathrm{~mm}$ diameter light beam from a $20 \mathrm{~mW} \mathrm{He}-\mathrm{Ne}$ laser (Meredith Instruments), modulated at $10 \mathrm{~Hz}$ by means of a mechanical chopper (Model 650 EG\&G), impinges on the black-painted outer surface of the $\mathrm{Al}$ foil. Following the absorption of the modulated light beam, the $\mathrm{Al}$ foil temperature fluctuates periodically at the modulation frequency of the incident beam thereby launching a thermal wave into the gas-filled cell. The thermal waves thus generated propagate back and forth between the $\mathrm{Al}$ foil and the pyroelectric sensor separated by a distance $L$. On striking the gas-Al and gas-sensor boundaries, the thermal waves are partially reflected, and interference between the reflected and incident wave trains will set in.

The temperature rise at the pyroelectric surface, which results from the superposition of all arriving waves, is probed using a lock-in amplifier (model 5210 EG\&G) interfaced to a personal computer. As discussed in Refs. 1-6, the method consists of measuring the temperature fluctuation at

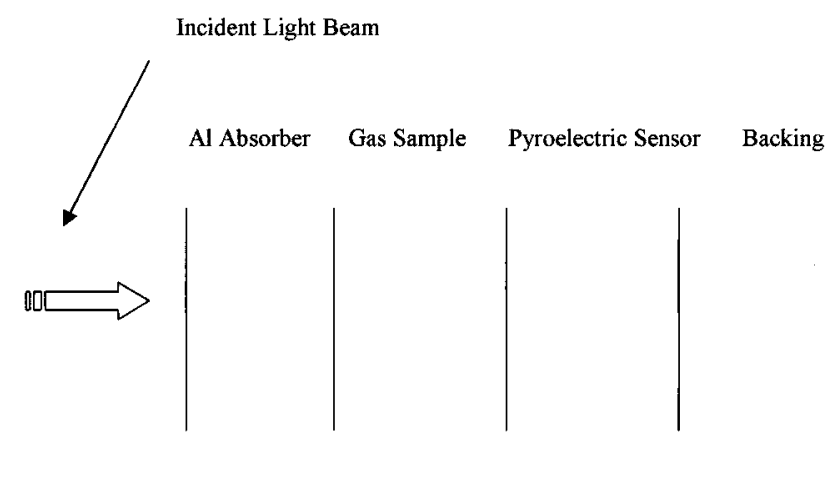

$\mathrm{x}=-\mathrm{L}_{0} \quad \mathrm{x}=0 \quad \mathrm{x}=\mathrm{L} \quad \mathrm{x}=\mathrm{L}+\mathrm{L}_{\mathrm{p}}$

FIG. 2. Geometry for the TWI signal generation in which the incident light beam of intensity $I_{0}$ is fully absorbed at $x=-L_{0}$.

the pyroelectric sensor as a function of the gas layer thickness. To this end the pyroelectric sensor is assembled on a micrometer stage coupled to a computer controlled stepping motor so that the cavity length can be varied. For a step resolution of $5 \mu \mathrm{m}$ the time required for the scanning of a 5 $\mathrm{mm}$ long cavity is roughly $4 \mathrm{~min}$.

The temperature rise at the sensor surface can be estimated using the usual thermal diffusion model for the generation of a photothermal signal. ${ }^{5-9}$ Consider the situation depicted in Fig. 2. A gas layer of thickness $L$ is sandwiched between a thermally thin $\left(L_{0} \sigma_{0} \ll 1\right) \mathrm{Al}$ foil and a pyroelectric sensor in contact with a thermally thick backing. The outer surface of the $\mathrm{Al}$ film is heated by a light source of periodically modulated intensity $I_{0}[1+\cos (\omega t)] / 2$, where $I_{0}$ is the light source intensity and $\omega=2 \pi f$ is the angular modulation frequency. The temperature distribution $T(x, t)$ within the gas region along the longitudinal $x$ coordinate, following the periodic heating of the $\mathrm{Al}$ foil at $x=-L_{0}$, can be obtained by solving the thermal diffusion equation with the boundary condition that the heat generated at the solid surface by light absorption is dissipated into the gas by diffusion. The laser beam is assumed to illuminate uniformly the $\mathrm{Al}$ foil in order to minimize the lateral heat diffusion effects. Under these conditions thermal gradients in the radial direction are negligible and the problem may be adequately treated as a one-dimensional problem. Performing lengthy but straightforward calculations, the harmonic component of the pyroelectric sensor surface temperature at $x$ $=L$ can be written as $T(L, t)=T(L) e^{j \omega t}$, where

$$
T(L)=\frac{\beta^{\prime} I_{0}}{\kappa_{0} \sigma_{0}} \cdot \frac{2}{s+\bar{p}} \cdot \frac{e^{-L \sigma}}{1-\gamma e^{-2 L \sigma}} .
$$

Here $\beta^{\prime}$ is the surface absorption coefficient, and $\sigma_{i}=(1$ $+j) / \mu_{i}$ is the complex thermal diffusion coefficient of medium $i$ with thermal diffusivity $\alpha_{i}$ and thermal effusivity $e_{i}$. The subscript $i=o, p$, and $b$ denote the $\mathrm{Al}$ foil light absorber, the pyroelectric sensor, and the backing materials, respectively. The thermal diffusivity and thermal effusivity are related to the thermal conductivity $\kappa$, specific heat $c$, and mass density $\rho$ by $\alpha=\kappa / \rho c$ and $e=(\kappa \rho c)^{1 / 2}$. The thermal diffu- 
sion length $\mu_{i}$ appearing in the expression for $\sigma_{i}$ is defined by $\mu_{i}=\left(\alpha_{i} / \pi f\right)^{1 / 2}$. In Eq. (1), $\gamma, \bar{p}$, and $s$ are defined as

$$
\gamma=\frac{s-\bar{p}}{s+\bar{p}}
$$

and

$$
\bar{p}=p \cdot\left(\frac{1-\left(\frac{p-b}{p+b}\right) \cdot e^{-2 L_{p} \sigma_{p}}}{1+\left(\frac{p-b}{p+b}\right) \cdot e^{-2 L_{p} \sigma_{p}}}\right) .
$$

Here $s=e / e_{0}, p=e_{p} / e_{0}$, and $b=e_{b} / e_{0}$ are the ratio of the thermal effusivities of the sample gas, pyroelectric sensor, and backing material with respect to $\mathrm{Al}$ foil thermal effusivity $e_{0}$, respectively. We refer to Ref. 9 for a more detailed discussion on thermal wave interference. Since the proposed TWI method consists basically of a recording sensor signal as a function of the gas layer thickness, the relevant factor in Eq. (1) is the last one on its right-hand side.

The temperature rise given by Eq. (1) induces an electric field between the two opposite surfaces of the PVDF sensor. The resulting voltage $V$ is proportional to the average temperature rise in the pyroelectric sensor. For a thermally thick pyroelectric sensor, as in our case, this is proportional to $T(L)$, so that the resulting measurable voltage may be written as

$$
V=V_{0} \cdot \frac{e^{-L \sigma}}{1-\gamma e^{-2 L \sigma}},
$$

where $V_{0}$ is a complex function containing the frequency response of the pyroelectric sensor signal and geometrical parameters characterizing the detection system. Here we note that the pyroelectric sensor output voltage depends explicitly on the modulation frequency and the cavity length $L$. The basic idea of the steady-state TWI technique consists of using a lock-in detection technique to recording the real (inphase) and imaginary (quadrature) parts of $V$, or, equivalently, its amplitude and phase, as a function of the cavity length $L$. From the resulting dependence of the pyroelectric signal voltage on the cavity length the thermal diffusivity is obtained from two different methods. One of them is based upon the fact that the $L$ dependence of Eq. (4) is such that their in-phase (IP) and quadrature $(Q)$ signals exhibit maxima and minima such that their $n$th extrema are given by $^{3,4}$

$$
L_{n}^{(\mathrm{IP})}=(n-1) \cdot\left(\frac{\alpha}{\pi f}\right)^{1 / 2}, \quad L_{n}^{(Q)}=n \cdot\left(\frac{\alpha}{\pi f}\right)^{1 / 2}
$$

with $n=1,2,3, \ldots$. That is, for a fixed modulation frequency, the positions of the maxima and minima of the in-phase and quadrature signals depend only on the thermal diffusivity $\alpha$ of the gas under investigation. The other method ${ }^{5-7}$ consists simply of fitting the recorded signal amplitude as a function of $L$ to Eq. (4), using standard data fitting procedures.

So far we have been discussing the TWI measurements under steady-state conditions. In the next section we address ourselves to the quantitative description of transient TWI measurements on air:hydrocarbon mixtures.

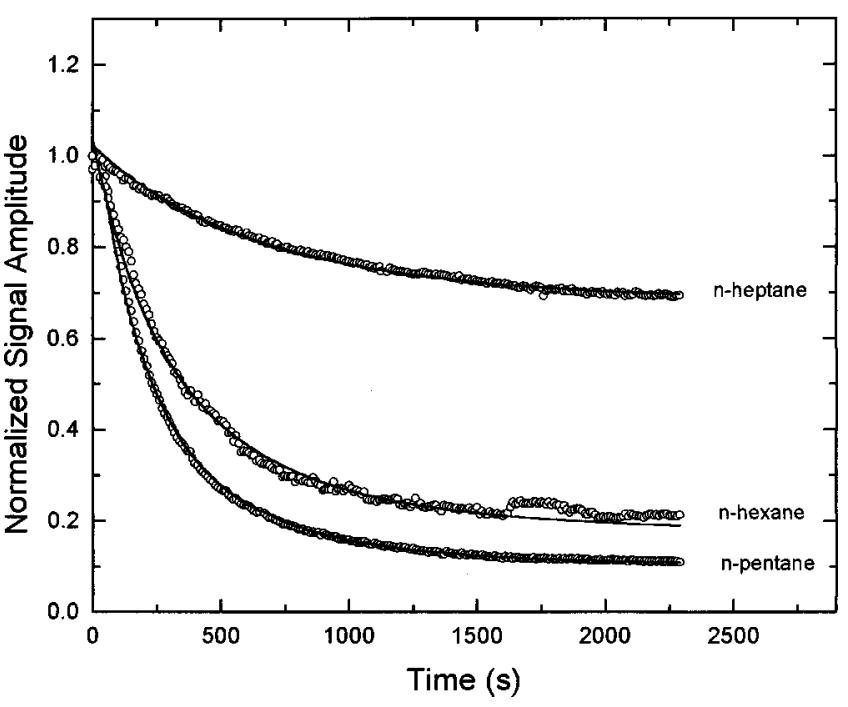

FIG. 3. Time evolution of the room temperature TWI signal amplitude, normalized to its initial $t=0$ value, of the different hydrocarbon samples. The corresponding signals were recorded at $10 \mathrm{~Hz}$ modulation frequencies with the TWI cavity length fixed at $2 \mathrm{~mm}$, at $23^{\circ} \mathrm{C}$. The solid curves in this plot correspond to the experimental data fitting of the following expression: $V_{N}=\exp \left\{-L\left(\pi f / \alpha_{\text {air }}\right)^{1 / 2}\left[\left(\alpha / \alpha_{\text {air }}\right)^{1 / 2}-1\right]\right\}$.

\section{TRANSIENT MEASUREMENTS: RESULTS AND DISCUSSION}

In Fig. 3 we show a typical time evolution of the TWI signal amplitude for different air:hydrocarbon mixtures recorded at $10 \mathrm{~Hz}$ modulation frequency for a fixed cavity length of $L=2 \mathrm{~mm}$. These measurements were carried out at an ambient temperature $\left(23^{\circ} \mathrm{C}\right)$ and pressure $(760 \mathrm{~mm} \mathrm{Hg})$ with the laboratory relative humidity at $60 \%$. As mentioned before, the experimental procedure consisted of pouring 18 $\mathrm{ml}$ of the liquid hydrocarbon at the bottom liquid reservoir of the TWI cell shown in Fig. 1, and recording the amplitude and phase of the TWI signal as a function of time. From the qualitative point of view, the signal decay as a function of time is reflecting the changes of the gas thermal diffusivity with time as a result of the changes of the hydrocarbon vapor concentration in air until a saturated mixture is formed. For the experimental situation in which the cavity length is fixed at $2 \mathrm{~mm}$ and modulation frequency is fixed at $10 \mathrm{~Hz}$, the gas layer in our TWI cavity may be considered as thermally thick so that Eq. (4) reduces to

$$
V=V_{0} \cdot e^{-L / \mu}=V_{0} \cdot e^{-L(\pi f / \alpha)^{1 / 2}} .
$$

It follows from Eq. (5) that decreasing the thermal diffusivity, as a result of the decrease of the gas thermal diffusivity due to the hydrocarbon vapor diffusion into the originally air-filled cell, the TWI signal decreases exponentially.

To quantitatively describe the behavior of the experimental data shown in Fig. 3 we assume that the TWI is adequately described by Eq. (5) in which the thermal diffusivity is a time-dependent function of the hydrocarbon concentration in the air. To model the time dependence of the thermal diffusivity we resort to the kinetic theory of gas mixtures. ${ }^{10,11}$ Due to the complexity of the theoretical expression for the thermal conductivity predicted by the kinetic 
TABLE I. Room temperature values of the thermal conductivity $(\kappa)$, heat capacity per unit volume $(\rho c)$, and thermal diffusivity $(\alpha)$ of the gases used in this work, taken from Refs. 16 and 17. Also included in the last two columns are the values of the hydrocarbon to air thermal conductivity and volume heat capacity ratio parameters, $\lambda$ and $\zeta$, respectively.

\begin{tabular}{cccccc}
\hline \hline Gas & $\kappa(\mathrm{mW} / \mathrm{cm} \mathrm{K})$ & $\rho c\left(\mathrm{~mJ} / \mathrm{cm}^{3} \mathrm{~K}\right)$ & $\alpha\left(\mathrm{cm}^{2} / \mathrm{s}\right)$ & $\lambda=\kappa / \kappa_{\text {air }}$ & $\zeta=(\rho c) /(\rho c)_{\text {air }}$ \\
\hline Air & 0.26 & 1.18 & 0.22 & 1 & 1 \\
$n$-pentane & 0.144 & 4.92 & 0.0293 & 0.554 & 4.17 \\
$n$-hexane & 0.134 & 5.86 & 0.0229 & 0.515 & 4.97 \\
$n$-heptane & 0.126 & 6.80 & 0.0185 & 0.485 & 5.76 \\
\hline \hline
\end{tabular}

theory, empirical expressions are often used to analyze the experimental data. Amongst these, the most widely used is the so-called logarithm mixing ${ }^{12}$ for the thermal conductivity. In this model the thermal conductivity for the binary mixture of components 1 and 2 is written as

$$
\kappa=\kappa_{1}^{(1-\eta)} \cdot \kappa_{2}^{\eta},
$$

where $\eta$ denotes the concentration of gas 2 (hydrocarbon vapor) in the reference gas 1 (air). As discussed in Ref. 12, the logarithm-mixing model for the thermal conductivity is quite adequate for the case of a random distribution of a two-phase system. Combining the effective thermal conductivity with the expression for the heat capacity per unit volume for the binary mixture, namely,

$$
\rho c=(\rho c)_{1} \cdot(1-\eta)+(\rho c)_{2} \eta,
$$

we can readily express the thermal diffusivity of the binary gas mixture in terms of the thermal properties of the constituent gases. One gets:

$$
\alpha=\alpha_{1} \cdot \frac{\lambda^{\eta}}{[1+(\zeta-1) \cdot \eta]},
$$

where we have introduced the parameters $\lambda$ and $\zeta$ defined as

$$
\lambda=\frac{\kappa_{2}}{\kappa_{1}}, \quad \zeta=\frac{(\rho c)_{2}}{(\rho c)_{1}} .
$$

Equation (8) is the basic equation we assume to describe the transient behavior of our air:hydrocarbon mixtures. The thermal diffusivity time dependence comes from the fact that the hydrocarbon concentration in the air-filled TWI cell varies with time until saturation is reached. Here we note that the extrapolation of the results from the heat diffusion with a constant $\alpha$ to transient measurements, implicit in Eq. (8), assumes a quasi-equilibrium approximation, namely, that the measurement time, at each cavity length, is shorter than the time-constant for the change in $\alpha$. Furthermore, the time dependent hydrocarbon concentration $\eta$ in the above random mixture model is to be understood as the spatially averaged hydrocarbon concentration in the gas cell.

To model the time evolution of the hydrocarbon concentration in the TWI cell we assume the simple Fick's model for mass diffusion of hydrocarbon vapor in the stagnant air of the TWI cell. For the sake of simplicity we assume that the relevant part of the diffusion process sensed by the TWI cavity corresponds to the hydrocarbon diffusion along the air column above the liquid reservoir shown in Fig. 1. Denoting by $z$ the coordinate along this gas column, and assuming a
Fickian diffusion, the equation describing the hydrocarbon vapor diffusion in the TWI cell may be written as

$$
\frac{\partial \eta}{\partial t}=D \frac{\partial^{2} \eta}{\partial z^{2}},
$$

where $D$ is the mass diffusion coefficient (mass diffusivity) of the hydrocarbon in air. The solution to the mass diffusion equation is discussed in several texts. ${ }^{13-15}$ Solving Eq. (10) for the gas column above the liquid reservoir of Fig. 1, with the boundary condition that the particle flux, $-D \partial \eta / \partial z$, at the bottom of the gas column is proportional to the concentration difference $\eta_{0}-\eta$, where $\eta_{0}$ is the hydrocarbon vapor saturation concentration, we may write the spatially averaged hydrocarbon concentration as

$$
\eta(t)=\eta_{0} \cdot\left(1-e^{-t / \tau}\right),
$$

where $\tau$ is the hydrocarbon vapor diffusion time in our system, defined as $\tau=L_{c}^{2} / 2 D$, in which $L_{c}$ is the characteristic length of our gas column. The derivation of Eq. (11) is presented in the Appendix. For the geometry of our TWI cell shown in Fig. 1, $L_{c}$ corresponds to the vertical gas column extending from the liquid level to the top of the glass cell and is equal to $10 \mathrm{~cm}$. Equations (8)-(11) together with Eq. (5) are the ones we shall use to describe the transient behavior of our TWI measurements.

To test the above model for the description of the transient TWI behavior we have applied it to the different set of measurements shown in Fig. 1. The solid curves in Fig. 3 represent the results of the normalized (to its initial $t=0$ value) TWI signal amplitude data fitting to the theoretical expression,

$$
V_{N}=e^{-L\left(\pi f / \alpha_{\mathrm{air}}\right)^{1 / 2} \cdot\left[\left(\alpha_{\mathrm{air}} / \alpha\right)^{1 / 2}-1\right],}
$$

in which the time dependent thermal diffusivity $\alpha$ is given by Eq. (8) with $\eta$ given by Eq. (11). Here we note that at $t$ $=0$ the TWI signal is simply that of an air-filled cavity. In carrying out the data fitting to Eq. (12) the only parameters left as adjustable parameters in the data fitting procedure were $\eta_{0}$ and $\tau$. All the other physical properties values involved in Eq. (8), namely, thermal conductivity and heat capacity per unit volume for the hydrocarbon and air, were taken from the literature and are summarized in Table I. For the room temperature signal amplitude data shown in Fig. 3, the values we got for the diffusion time $\tau$ from the data fitting were $580.8187 \pm 2.5061 \mathrm{~s}, 701.5513 \pm 5.3165 \mathrm{~s}$, and $793.0834 \pm 3.5469 \mathrm{~s}$, for $n$-pentane, $n$-hexane, and $n$-heptane, respectively. From these values of $\tau$, and remembering that 
TABLE II. Values of the fitting parameters $\eta_{0}$ and $\tau$ as obtained from the room temperature TWI signal amplitude data fitting. The values of the hydrocarbons diffusion coefficients in air, $D$, were calculated from $D=L_{c}^{2} / 2 \tau$, where $L_{c}$ was taken to be equal to the length of the gas column above the liquid reservoir, namely, $L_{c}=10 \mathrm{~cm}$. The literature values of the hydrocarbon diffusion coefficients in air, listed in the last column, were taken from Ref. 18.

\begin{tabular}{lcccc}
\hline \hline Hydrocarbon & $\eta_{0}$ & $\tau(\mathrm{s})$ & $D\left(\mathrm{~cm}^{2} / \mathrm{s}\right)$ & $D_{\text {literature }}\left(\mathrm{cm}^{2} / \mathrm{s}\right)$ \\
\hline$n$-pentane & $0.534 \pm 0.002$ & $580.8187 \pm 2.5061$ & $0.0861 \pm 0.0004$ & 0.0842 \\
$n$-hexane & $0.349 \pm 0.001$ & $701.5513 \pm 5.3165$ & $0.0713 \pm 0.0006$ & 0.0732 \\
$n$-heptane & $0.062 \pm 0.0001$ & $793.0834 \pm 3.54688$ & $0.0630 \pm 0.0002$ & 0.0674 \\
\hline \hline
\end{tabular}

the characteristic length $L_{c}$ is equal to $10 \mathrm{~cm}$, we can estimate the corresponding hydrocarbon diffusion coefficient in air from $D=L_{c}^{2} / 2 \tau$. For instance, for $n$-pentane the value we got for $D$ was $0.0861 \pm 0.0004 \mathrm{~cm}^{2} / \mathrm{s}$. This value is in good agreement with the one reported in the literature ${ }^{18}$ for $n$-pentane, namely, $D=0.0842 \mathrm{~cm}^{2} / \mathrm{s}$. The same procedure was adopted with the other two hydrocarbons investigated. In Table II we summarize the results of our TWI signal amplitude data fitting; also included are the values of the mass diffusion coefficient for the different hydrocarbons reported in the literature. These results indicate that the above model provides an adequate description of the transient TWI signal behavior.

\section{CONCLUSIONS}

In this article we have addressed ourselves to the quantitative description of the TWI monitoring of the transients of hydrocarbon the evaporation kinetics in air. This was carried out with a TWI cell at fixed modulation frequency and cavity length, and recording the corresponding signal amplitude as a function of time. The TWI signal was modeled assuming the logarithm-mixing model for the hydrocarbon vapor:air mixture in which the hydrocarbon vapor concentration is a function of time. The time evolution of the hydrocarbon vapor concentration in the air-filled TWI cell was described assuming the simple Fick's model for mass diffusion of the hydrocarbon vapor in the stagnant air column of the TWI cell. The transient TWI signal amplitude data fitting yielded two parameters, namely, the saturation concentration $\eta_{0}$ and the characteristic diffusion time $\tau$. From the corresponding values of $\tau$, the hydrocarbon mass diffusivities were straightforwardly obtained. The obtained values for the hydrocarbon mass diffusivities were found to be in good agreement with the ones reported in the literature thereby validating the proposed description of the TWI monitoring of hydrocarbon evaporation kinetics.

The results presented in this article not only fill the lack of discussion on the transient TWI signal description but also suggest a framework for investigating a wide range of applications involving transient diffusion, such as the monitoring of soil and plants respiration kinetics and pollutant diffusion in ambient air.

\section{ACKNOWLEDGMENT}

The authors are grateful to the Brazilian agencies CNPq, ANP, and Faperj for the partial support of this work.

\section{APPENDIX}

Equation (10) describes the hydrocarbon vapor diffusion along the gas column of the TWI cell. The parameter $D$ in this equation is the mass diffusion coefficient of the hydrocarbon vapor in air at room temperature. For a given chemical specie diffusing in air it is assumed to be a characteristic constant value. To solve Eq. (10) we first take its Laplace transform, in which case it is written as

$$
\frac{\partial^{2} \tilde{\eta}}{\partial z^{2}}=\frac{\nu}{D} \tilde{\eta},
$$

where $\tilde{\eta}$ is the Laplace transform of $\eta$, and $\nu$ is the (frequency) variable of the Laplace space. The solution to the Laplace transformed one-dimensional mass diffusion equation can be written as

$$
\tilde{\eta}(z, \nu)=A e^{\sigma z}+B e^{-\sigma z}
$$

where $\sigma=(\nu / D) \frac{1}{2}, A$ and $B$ are constants to be determined by the boundary conditions imposed on our problem. In our case, the boundary conditions are such that at the liquid reservoir:gas interface at $z=0$, the hydrocarbon particle flux into the TWI cell, $-D \partial \tilde{\eta} / \partial z$, is proportional to the concentration difference $\eta-\eta_{0}$, where $\eta_{0}$ is the final hydrocarbon vapor saturation concentration. The other boundary condition is that at the end of the TWI cell there is no particle flux, namely, $D \partial \tilde{\eta} / \partial z=0$ at $z=L_{c}$. In this way, our problem is then reduced to find $A$ and $B$ such that

$$
-D\left(\frac{\partial \tilde{\eta}}{\partial z}\right)_{z=0}=k\left(\frac{\eta_{0}}{V}-\tilde{\eta}\right)_{z=0}\left(\frac{\partial \tilde{\eta}}{\partial z}\right)_{z=L_{c}}=0
$$

where $k$ is a proportionality constant. Substituting Eq. (A2) into Eq. (A3) one finds:

$$
\begin{aligned}
& A=\frac{h \eta_{0}}{\nu} \frac{e^{-L_{c} \sigma}}{(1+h) e^{L_{c} \sigma}-(1-h) e^{-L_{c} \sigma}}, \\
& B=\frac{h \eta_{0}}{\nu} \frac{e^{L_{c} \sigma}}{(1+h) e^{L_{c} \sigma}-(1-h) e^{-L_{c} \sigma}},
\end{aligned}
$$

where $h=k / D \sigma$. The spatially averaged concentration $\langle\widetilde{\eta}(\nu)\rangle$ is obtained from

$$
\begin{aligned}
\langle\tilde{\eta}(\nu)\rangle & =\frac{1}{L_{c}} \int_{0}^{L_{c}} d z \tilde{\eta}(z, \nu) \\
& =\frac{1}{L_{c} \sigma} \frac{h \eta_{0}}{\nu} \frac{e^{L_{c} \sigma}-e^{-L_{c} \sigma}}{(1+h) e^{L_{c} \sigma}-(1-h) e^{-L_{c} \sigma}} .
\end{aligned}
$$

At long observation times, $t \rightarrow \infty, \nu \rightarrow 0$, Eq. (A5) reduces to 


$$
\langle\tilde{\eta}(\nu)\rangle=\eta_{0} \frac{1}{\nu(1+\nu \tau)},
$$

where $\tau=L_{c}^{2} / 2 D$ is the characteristic mass diffusion time. Taking the inverse Laplace transform of Eq. (A6) one may finally write the spatially averaged time-dependent hydrocarbon vapor concentration as

$$
\eta(t)=\eta_{0}\left(1-e^{-t / \tau}\right) .
$$

${ }^{1}$ C. A. Bennett and R. R. Patty, Appl. Opt. 21, 49 (1982).

${ }^{2}$ J. Shen and A. Mandelis, Rev. Sci. Instrum. 66, 4999 (1995).

${ }^{3}$ J. Shen, A. Mandelis, and B. D. Aloysius, Int. J. Thermophys. 17, 1241 (1996).

${ }^{4}$ J. Shen, A. Mandelis, and T. Ashe, Int. J. Thermophys. 19, 579 (1998).

${ }^{5}$ J. A. P. Lima, E. Marin, M. G. Silva, M. S. Sthel, S. L. Cardoso, D. F.

Takeuti, C. Gatts, H. Vargas, C. E. Rezende, and L. C. M. Miranda, Rev.

Sci. Instrum. 71, 2928 (2000)

${ }^{6}$ J. A. P. Lima, E. Marin, M. G. Silva, M. S. Sthel, S. L. Cardoso, H.
Vargas, and L. C. M. Miranda, Rev. Sci. Instrum. 72, 1 (2001).

${ }^{7}$ J. A. P. Lima, E. Marin, O. Correa, M. G. Silva, S. L. Cardoso, C. Gatts, C. E. Rezende, H. Vargas, and L. C. M. Miranda, Meas. Sci. Technol. 11, $1522(2000)$

${ }^{8}$ H. Vargas and L. C. M. Miranda, Phys. Rep. 161, 43 (1988).

${ }^{9}$ D. Almond and J. Patel, Photothermal Science and Technology (Chapman and Hall, London, 1996).

${ }^{10}$ J. O. Hirschfelder, C. F. Curtiss, and R. B. Bird, Molecular Theory of Gases and Liquids (Wiley, New York, 1964).

${ }^{11}$ N. B. Srivastava and S. C. Saxena, Phys. Fluids 27, 583 (1957).

${ }^{12}$ R. P. Tye, Thermal Conductivity (Academic, New York, 1969), Vol. 1.

${ }^{13}$ J. Crank, The Mathematics of Diffusion (Clarendon, Oxford, 1975).

${ }^{14}$ E. L. Cussler, Multicomponent Diffusion (Elsevier, New York, 1976).

${ }^{15}$ P. Neogi, Diffusion in Polymers (Marcel Dekker, New York, 1996).

${ }^{16}$ D. Lyde, CRC Handbook of Chemistry and Physics, 75th ed. (Chemical Rubber Co., Cleveland, 1995).

${ }^{17}$ L. E. Sissom and D. R. Pitts, Elements of Transport Phenomena (McGraw-Hill, New York, 1972).

${ }^{18}$ C. L. Yaws, Handbook of Transport Properties Data: Viscosity, Thermal Conductivity and Diffusion Coefficients (Gulf, Houston, 1995). 